\title{
Enhancement of BOLD-Contrast Sensitivity by Single-Shot Multi-Echo Functional MR Imaging
}

\author{
Stefan Posse, ${ }^{1 *}$ Stefan Wiese, ${ }^{1}$ Daniel Gembris, ${ }^{1}$ Klaus Mathiak, ${ }^{1}$ Christoph Kessler; ${ }^{2}$ \\ Maria-Liisa Grosse-Ruyken, ${ }^{1}$ Barbara Elghahwagi, ${ }^{1}$ Todd Richards, ${ }^{3}$ Stephen R. Dager, ${ }^{3}$ \\ and Valerij G. Kiselev ${ }^{1}$
}

Improved data acquisition and processing strategies for blood oxygenation level-dependent (BOLD)-contrast functional magnetic resonance imaging ( $\mathrm{FMRI}$ ), which enhance the functional contrast-to-noise ratio (CNR) by sampling multiple echo times in a single shot, are described. The dependence of the CNR on $T_{2}^{*}$, the image encoding time, and the number of sampled echo times are investigated for exponential fitting, echo summation, weighted echo summation, and averaging of correlation maps obtained at different echo times. The method is validated in vivo using visual stimulation and turbo proton echoplanar spectroscopic imaging (turbo-PEPSI), a new single-shot multi-slice MR spectroscopic imaging technique, which acquires up to 12 consecutive echoplanar images with echo times ranging from 12 to $213 \mathrm{msec}$. Quantitative $\mathrm{T}_{2}^{*}$-mapping significantly increases the measured extent of activation and the mean correlation coefficient compared with conventional echoplanar imaging. The sensitivity gain with echo summation, which is computationally efficient provides similar sensitivity as fitting. For all data processing methods sensitivity is optimum when echo times up to $3.2 \mathrm{~T}_{2}^{*}$ are sampled. This methodology has implications for comparing functional sensitivity at different magnetic field strengths and between brain regions with different magnetic field inhomogeneities. Magn Reson Med 42:87-97, 1999. 1999 Wiley-Liss, Inc. Key words: fMRI; BOLD contrast; MR; sensitivity; multi-echo; spectroscopic imaging

Functional MR imaging (fMRI) is a powerful emerging tool for mapping brain activation with high spatial and temporal resolution. However, fMRI suffers from a number of limitations as follows:

1. Sensitivity constraints at clinical field strengths limit studies of higher cognitive functions. In many cases multi-subject averaging is necessary, despite the wellrecognized limitations of that approach.

2. Absolute quantitation of functional signal changes, which exhibit between-subject and within-subject variability, is not yet feasible. This limitation is in part due to poorly characterized signal contributions from the microvasculature, large blood vessels, and cerebrospinal fluid (CSF).

3. Conventional blood oxygenation level-dependent (BOLD)-contrast fMRI techniques measure the signa

IInstitute of Medicine, Research Center Jülich GmbH, Jülich, Germany. 'Department of Psychiatry, University of Düsseldorf, Düsseldorf, Germany. ${ }^{3}$ Departments of Radiology and Bioengineering, University of Washington, Seattle, Washington.

Grant sponsor: EU, Biomed II; Grant number: 950870

'Correspondence to: Stefan Posse, Institute of Medicine, Research Center Jülich $\mathrm{GmbH}$, Leo-Brandt-Strasse, D-52425 Jülich, Germany.

Received 14 August 1998; revised 21 December 1998; accepted 15 March 1999 .

o 1999 Wiley-Liss, Inc. decay at a single echo time, which closely matches the average tissue $T_{2}^{*}$ to optimize functional contrast. However, regional and inter-individual variations in intrinsic tissue $T_{2}^{*}$, hardware instabilities, as well as possible changes in initial signal intensity $\left(\mathrm{S}_{\mathrm{o}}\right)$ due to flow effects, confound quantitation of the observed signal changes.

Recent advances in characterizing the BOLD-contrast mechanism and the spatial localization of functional signal changes during neuronal activation were made possible by acquisition techniques that sample multiple time points of the water relaxation decay $(1-18)$. Furthermore, distinction of $\mathrm{T}_{2}^{*}$-relaxation and other factors (e.g., inflow, hardware instabilities) can be achieved (9). In previous fMRI studies, the BOLD-contrast signal decay curve in gray matter has been shown to be approximately monoexponential between echo times of 30 and $100 \mathrm{msec}(1,4,6,8)$. In and adjacent to blood vessels, multiexponential decay has been found (6). To quantitate functional relaxation changes using conventional fMRI techniques [fast low-angle shot (FLASH)/gradient-recalled acquisition in a steady-state pulse (GRASS) or echoplanar imaging (EPI)], it is necessary to repeat measurements with different echo times. However, such measurements are very time consuming due to the many repetitions of the stimulation paradigm at different echo times, which makes the results sensitive to physiological variability (e.g., from changes in attention) during the measurements and introduces sensitivity to head motion artifacts. This limits practical measurements to only a few time points along the signal decay curve. A significant reduction of the measurement time is possible by multiecho (spectroscopic) imaging methods that measure functional signal changes at multiple echo times in a single excitation $(1,3,4,6,7-14)$. Recently, we have used fast spectroscopic imaging based on our proton echoplanar spectroscopic imaging (PEPSI) method $(15,16)$ to map functional changes in water $\mathrm{T}_{2}^{*}$ quantitatively during visual stimulation (9). However, that technique was limited to a single slice and had poor temporal resolution of 4.5 sec. The most advanced methods can now measure complete two-dimensional (2D) relaxation maps in a single shot (11-14).

An alternative method with much finer sampling of the signal decay curve has been developed by Hennig et al (17), who employed PRESS single-voxel spectroscopy to quantitate changes in $\mathrm{T}_{2}^{*}$ during visual stimulation. In a subsequent study, the high intrinsic sensitivity of the method enabled them to detect subtle signal changes associated with the so-called negative dip during the first second of activation (18). However, this single-voxel technique is not 
suitable for mapping the spatial distribution of the BOLDcontrast signal changes, and its spatial resolution is very coarse compared with that of fMRI.

To our knowledge, the sensitivity of multiecho fMRI methods to changes in BOLD relaxation has not yet been systematically characterized in comparison with conven tional single-echo techniques. The BOLD effect, which is most conveniently viewed as a bell-shaped difference signal between two relaxation curves measured during activation and during baseline, has a maximum at a time delay that corresponds approximately to the mean of the $\mathrm{T}_{2}^{*}$ values of the two curves (Fig. 1). The time course of the difference signal suggests that to maximize sensitivity in the presence of noise it is advantageous to use a sampling window that encompasses the major part of the bellshaped curve. This can be achieved either by using EPI with very low detection bandwidths (which, however, increases geometrical image distortions) or by repeating the same EPI encoding multiple times in a single shot at a correspondingly higher detection bandwidth.

In this paper we assess the dependence of the contrast-tonoise ratio (CNR) of single-shot multiecho EPI data sets on $\mathrm{T}_{2}^{*}$, the $k$-space encoding speed, the spatial resolution, and the number of echoes acquired. To validate our findings in vivo, we used a new single-shot multislice $T_{2}^{*}$-sensitive MR spectroscopic imaging method (turbo-PEPSI), which acquires 12 consecutive echoplanar images with equidistant echo times ranging from 12 to $213 \mathrm{msec}$ with a spectral bandwidth of $55 \mathrm{~Hz}$. Since curve fitting of the echo data is time consuming, faster data-processing methods were also analyzed, comprising linear and weighted summation of data acquired at different echo times and averaging of correlation maps obtained at different echo times. We have previously reported preliminary results in abstract form $(12,14)$.

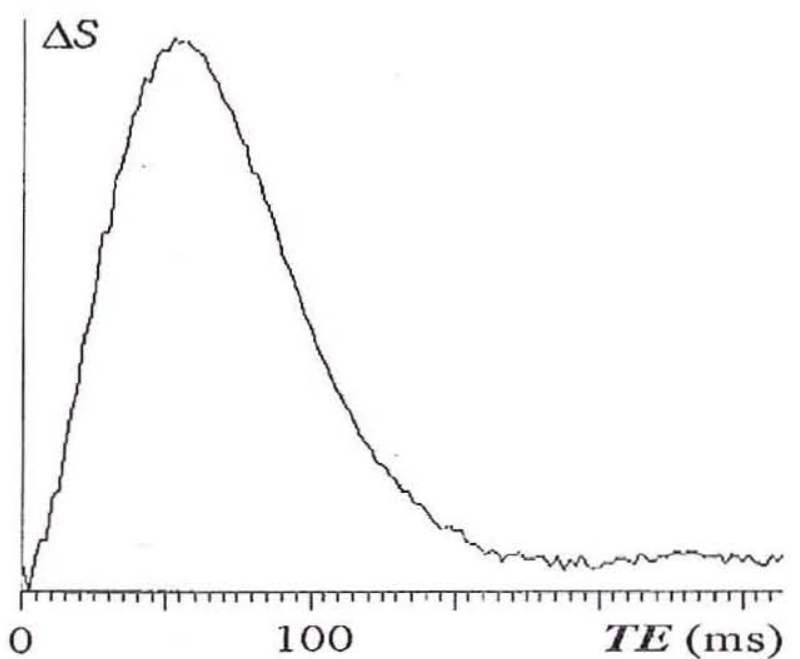

FIG. 1. Typical BOLD contrast difference signal $(8 \mathrm{~Hz}$ flicker light activation-baseline) in a central voxel in visual cortex measured with the PEPSI spectroscopic imaging method (TE 2.5-258.5 msec, TR $293 \mathrm{msec} \alpha 35^{\circ}$, voxel size $\left.6 \times 6 \times 6 \mathrm{~mm}^{3}\right)$. The maximum difference signal in that study (9) was observed at a TE of $69 \pm 5$ msec.

\section{THEORY}

\section{Data-Processing Strategies}

Let us assume that the stimulation paradigm is measured at time points $t_{\mathrm{r}}$ (measurement time domain) using a singleshot multiecho measurement at echo times $T E_{n}$ (echo time domain) with an interecho spacing $\Delta t$, which is identical to the image encoding time and inversely proportional to the receiver bandwidth. In the context of spectroscopic imaging the inverse of the interecho spacing is commonly referred to as the spectral bandwidth $(9,15,16)$. Assuming monoexponential relaxation, the multiecho signal in a given voxel can be expressed as:

$$
S\left(t_{r}, T E_{n}\right)=S_{0}\left(t_{r}\right) \exp \left(-\frac{T E_{n}}{T_{2}^{*}\left(t_{r}\right)}\right)+g_{n r}+h_{r}
$$

where the subscript $r=1, \ldots, M$ labels the repetitions of the multiecho measurement and $n=1, \ldots, N$ labels the echo number. $S_{0}\left(t_{r}\right)$ is the initial signal amplitude, which may vary from measurement to measurement due to hardware instabilities or flow-related saturation effects. $\mathrm{T}_{2}^{*}\left(t_{t}\right)$ is the stimulus-dependent relaxation time. Signal fluctuations are assumed to consist of two components: white noise $g_{n r}$ (thermal noise and hardware instabilities) and more slowly varying noise $h_{r}$, which reflects physiological mechanisms, such as heart-beat-related brain pulsation and stimulusindependent vasomotor activity (19). The noise $g_{n r}$ is white at least up to the frequency $1 / \Delta t$ and depends on imaging parameters such as voxel size and receiver bandwidth. This noise has zero mean and a standard deviation $\sigma_{g}$, which is independent of $T E_{n}$ and $t_{r}$. The physiological noise $h_{r}$ describes fluctuations in $S_{0}\left(t_{r}\right)$ and $\mathrm{T}_{2}^{*}\left(t_{r}\right)$ in the domain of repetition time. The value of $h_{r}$ is constant during a single multiecho measurement. This noise has zero mean and statistical deviation $\sigma_{\mathrm{h}}$ which is TE independent. For simplicity, we assume Gaussian noise distributions and statistical independence of the two noise components.

The BOLD-contrast difference signal $\Delta S$ between the activated state and the baseline state can be found by assuming small changes in $\mathrm{T}_{2}^{*}\left(t_{r}\right)$ :

$$
T_{2}^{*}\left(t_{r}\right)=T_{2}^{*}+\Delta T_{2}^{*}\left(t_{r}\right), \quad \Delta T_{2}^{*}\left(t_{r}\right) \ll T_{2}^{*}
$$

and by expanding in $\Delta \mathrm{T}_{2}^{*}\left(t_{\mathrm{r}}\right)$ :

$$
\begin{aligned}
\Delta S\left(t_{r}, T E_{n}\right) \approx S_{0} \Delta T_{2}^{\star}\left(t_{t}\right) \frac{d}{d T_{2}^{*}} \exp \left(-\frac{T E_{n}}{T_{2}^{*}}\right) \\
=S_{0} \frac{T E_{n}}{T_{2}^{*}} \exp \left(-\frac{T E_{n}}{T_{2}^{*}}\right) \frac{\Delta T_{2}^{*}\left(t_{t}\right)}{T_{2}^{*}}
\end{aligned}
$$

This bell-shaped curve (the contrast) has its maximum value

$$
\frac{S_{0} \Delta T_{2}^{*}\left(t_{r}\right)}{e T_{2}^{*}} \approx 0.37 \frac{S_{0} \Delta T_{2}^{*}\left(t_{r}\right)}{T_{2}^{*}}
$$

at $T E_{n}=\mathrm{T}_{2}^{*}$, which is typically $70 \mathrm{msec}$ at $1.5 \mathrm{~T}$ (Fig.1). With conventional fMRI techniques, only a single time 
point close to the expected BOLD effect maximum is measured. The image encoding time for a $64 \times 64$ spatial matrix is typically on the order of $40-60 \mathrm{msec}$. Longer measurement times (lower phase-encoding bandwidths) are rarely used due to the stronger sensitivity to geometrical distortions.

Under these conditions, additional functional information can be obtained by sampling multiple echo times in a single shot and by combining the data sets thus obtained. The degree of sensitivity enhancement is not only dependent on $\mathrm{T}_{2}^{*}$ and the image encoding time, but also on how the data sets are combined. Since the measured signals for each voxel form a $2 \mathrm{D}$ array in $r$ and $n$, the functional signal analysis can either start with the echo time domain and continue with the measurement repetition time domain or proceed in the reverse order, although more flexible 2D approaches may also be useful. When starting with the echo time domain, several data-processing options (1.-3.) are available:

1. Summation of data sets acquired at different echo times that is computationally fast yields a combined measure of $S_{0}$ and $\mathrm{T}_{2}^{*}$ :

$$
\hat{S}\left(t_{r}\right)=\sum_{n=1}^{N} S\left(t_{r}, T E_{n}\right)
$$

The disadvantage of this method is that noisy data sets measured at long echo times reduce the overall gain in sensitivity. The bell shape of the difference signal suggests that:

2. A weighted summation of the data sets acquired at different echo times increases sensitivity compared with simple summation:

$$
\hat{S}\left(t_{r}\right)=\sum_{n=1}^{N} S\left(t_{r}, T E_{n}\right) W\left(T E_{n}\right),
$$

where the weighting function:

$$
w\left(T E_{n}\right)=\frac{T E_{n}}{\tau} \exp \left(-\frac{T E_{n}}{\tau}\right)
$$

is employed. The time constant $\tau$ should match the expected relaxation time $\mathrm{T}_{2}^{*}$. This approach is similar to the matched filter used in spectroscopy. Unlike in spectroscopy, an exponentially decaying weighting function that matches the shape of the relaxation signal is less advantageous for measuring the difference signal. It is of interest to compare these approaches with:

3. Curve fitting in the time or the spectral domain to quantitate changes in $\mathrm{T}_{2}^{*}$ and $S_{0}$. Although this approach, which was the only method used in the past to analyze multiecho measurements, has the advantage of being quantitative, computational requirements may limit clinical applications.

After these preprocessing steps, algorithms for detect- ing neuronal activity (such as correlation analysis) can be applied.

4. When starting with the measurement time domain, statistical analysis is performed for each echo separately. The data can be processed like conventional fMRI data (e.g., by correlation analysis), and the resulting statistical maps can be combined to increase statistical significance, e.g., by averaging.

\section{Sensitivity Optimization}

A decision must be made as to how far to the left and to the right of the maximum of the difference signal sampling is necessary in order to optimize sensitivity. For simplicity, we assume equidistant sampling with an echo spacing $\Delta t$, which is equal to the image-encoding time, a minimum echo time of $\mathrm{TE}_{1}=\Delta t$, and a total sampling time $N \Delta t$. The dependence on the starting point at the left side of the maximum is not further considered, since it does not significantly modify the results due to the steep initial rise of the difference signal with increasing echo time. The CNR of the single-echo measurement, which serves as a reference for the sensitivity enhancement, is obtained from the contrast in Eq. [4]:

$$
C N R_{0}=\frac{0.37 \cdot S_{0} \Delta T_{2}^{*}\left(t_{t}\right)}{\sqrt{2} \sigma_{g} T_{2}^{*}}
$$

where $\sqrt{2} \sigma_{g}$ is the standard deviation of the white noise of the difference signal computed in Eq. [3].

1. For the case of simple summation of signals acquired at different echo times, the resulting difference of the activation and baseline signals can be expressed as:

$$
\begin{aligned}
& \sum_{n=1}^{N} \Delta S\left(t_{r}, n \Delta t\right) \\
& \quad=S_{0} e^{-\Delta U T_{2}^{*}} \frac{1-e^{-(N+1) \Delta U T_{2}^{*}}}{1-e^{-\Delta t / T_{2}^{*}}} \approx S_{0} \frac{T_{2}^{*}}{\Delta t}\left[1-e^{-N \Delta U T_{2}^{*}}\right]
\end{aligned}
$$

where $N>1$. As the BOLD effect provides a relatively small change in $T_{2}{ }^{*}$, the following approximation for the contrast $C$ can be made:

$$
\begin{aligned}
C=S_{0} \Delta T_{2}^{*}\left(t_{r}\right) \frac{d}{d T_{2}^{*}} \sum_{n=1}^{N} \Delta S\left(t_{r}, n \Delta t\right) \\
\\
\approx S_{0} \frac{\Delta T_{2}^{*}\left(t_{r}\right)}{\Delta t}\left[1-(x+1) e^{-x}\right]
\end{aligned}
$$

where $x \equiv N \Delta t / T_{2}^{*}$. The white noise in this summed up signal has the standard deviation $\sqrt{2 N} \sigma_{g}$. Thus, the CNR of a single multiecho measurement is:

$$
C N R_{N}=\frac{S_{0}}{\sqrt{2} \sigma_{g}} \frac{\Delta T_{2}^{*}}{T_{2}^{*}} \sqrt{\frac{T_{2}^{*}}{\Delta t}} \frac{\left[1-(x+1) e^{-x}\right]}{\sqrt{x}}
$$




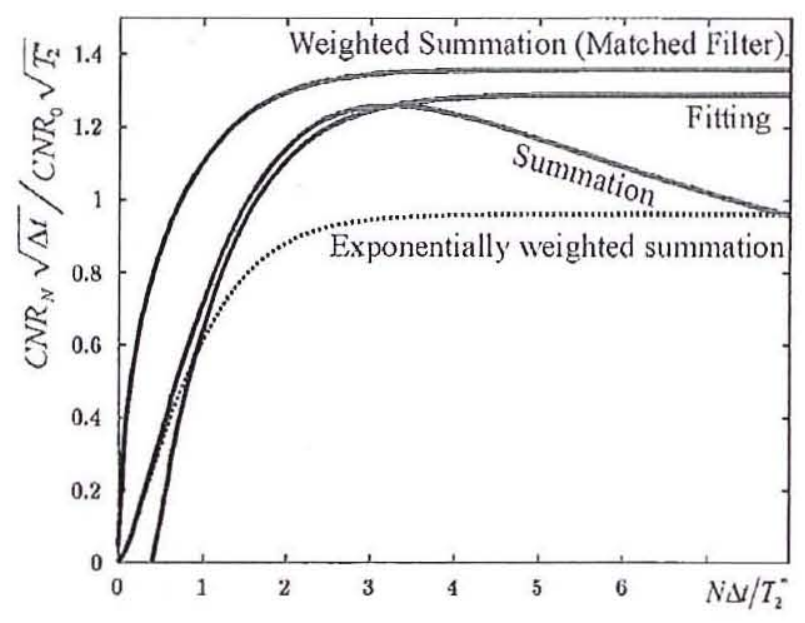

FIG. 2. Theoretical gain in contrast-to-noise ratio by multiecho $\left(C N R_{N}\right)$ compared with single-echo $\left(C N R_{0}\right)$ sampling for different data-processing methods in the echo time domain as a function of the number of measured echoes $(N)$, scaled by the intrinsic $T_{2}^{*}$-value and the measurement interval $\Delta t$. Exponentially weighted summation is less advantageous than simple summation and curve fitting. The best performance is predicted for weighted summation using a matched filter.

The $\mathrm{CNR}_{N}$ has a maximum at $x=3.2$ (Fig.2). Thus, sampling of the free induction decay (FID) should be performed with a total measurement time:

$$
T_{\text {opt }}=3.2 T_{2}^{*}
$$

which for a given image-encoding time $\Delta t$ corresponds to the optimum number of measured echo times:

$$
N_{\text {opt }} \approx 3.2 T_{2}^{*} / \Delta t
$$

For this $N_{\mathrm{opt}}$, the CNR is maximum and equal to:

$$
\begin{aligned}
& C N R_{N} \\
& \quad=0.46 \frac{S_{0}}{\sqrt{2} \sigma_{g}} \frac{\Delta T_{2}^{*}\left(t_{r}\right)}{T_{2}^{*}} \sqrt{\frac{T_{2}^{*}}{\Delta t}}=1.2 \sqrt{\frac{T_{2}^{*}}{\Delta t}} C N R_{0}
\end{aligned}
$$

For numerical estimates of sensitivity enhancement, we use $\mathrm{T}_{2}^{*}=70 \mathrm{msec}$ (at $1.5 \mathrm{~T}$ ) and compare an EPI encoding time $\Delta t$ of $50 \mathrm{msec}$, which is typical for many functional studies with $\Delta t=18.3 \mathrm{msec}$, which reflects our measurement conditions (see below). The optimum number of echoes for the two EPI encoding times are 4 and 12, respectively, with total sampling times of 200 and 220 msec. The gains in sensitivity compared with the corresponding single-echo measurements are 1.42- and 2.35-fold, respectively. Thus, even for $\Delta t=50 \mathrm{msec}$, a multiecho measurement results in significant gains in sensitivity.

2. For weighted summation using calculations analogous to those performed above, the following CNR was obtained (Fig. 2):

$C N R_{N}$

$$
=\frac{S_{0}}{\sqrt{2} \sigma} \frac{\Delta T_{2}^{*}}{T_{2}^{*}} \sqrt{\frac{T_{2}^{*}}{\Delta t}} \sqrt{\frac{2-\left(x^{2}+2 x+2\right) e^{-2 x}}{8}}
$$

For large $x$, this function has an asymptotic value:

$C N R_{N}$

$$
=\frac{S_{0}}{2 \sqrt{2} \sigma_{g}} \frac{\Delta T_{2}^{*}\left(t_{t}\right)}{T_{2}^{*}} \sqrt{\frac{T_{2}^{*}}{\Delta t}}=1.36 \sqrt{\frac{T_{2}^{*}}{\Delta t}} C N R_{1}
$$

while its value for the echo time $3.2 \mathrm{~T}_{2}^{*}$ is only $1 \%$ smaller (Fig. 2). The asymptotic value in Eq. [16] is approximately $13 \%$ higher than the value obtained for simple summation. By contrast, exponential weighting of the data is less advantageous, as shown in Fig. 2.

3. Curve fitting of the multiecho data corresponds to the minimization of:

$$
F=\frac{1}{2} \sum_{n}\left[S\left(t_{r}, T E_{n}\right)-f\left(\vec{x}_{,} t_{n}\right)\right]^{2}
$$

where $f$ describes the curve fit (e.g., a monoexponential decay). which is dependent on time and a set of parameters $\overrightarrow{\mathrm{x}}=\left(x_{1}, x_{2}, \ldots\right)$ (e.g., the initial signal magnitude and the relaxation rate). To assess the CNR of curve fitting, we expand $F$ in the difference $d S_{n}=S\left(t_{r}, T E_{n}\right)-f\left(\vec{x}, t_{n}\right)$ and in the variation of the parameters $d \vec{x}$ up to the second-order terms, which are predominant because the first-order terms are zero at the minimum of $F$ :

$$
\begin{aligned}
F \approx \sum_{n, l} \frac{\partial^{2} F}{\partial S_{n} \partial x_{i}} d S_{n} d x_{i} & +\frac{1}{2} \sum_{i, j} \frac{\partial^{2} F}{\partial x_{i} \partial x_{j}} d x_{i} d x_{j} \\
& +\frac{1}{2} \sum_{n, m} \frac{\partial^{2} F}{\partial S_{n} \partial S_{m}} d S_{n} d S_{m}
\end{aligned}
$$

The noise-dependent parameter variation can be found by the minimization of this quadratic form. The result is:

$$
d \vec{x}=-\left(\frac{\partial^{2} F}{\partial x_{i} \partial x_{j}}\right)^{-1}\left(\sum_{n} \frac{\partial^{2} F}{\partial S_{n} \partial x_{j}} d S_{n}\right)
$$

In this product of matrices, the inversion implies a matrix inversion of the dimension of $\vec{x}$, which is small. For the sake of illustration, we perform the explicit calculations in Eq. [19] for fitting $\mathrm{T}_{2}^{*}$ with constant $S_{0}$. The estimate we obtain for the CNR is shown in Fig. 2.

4. When starting the correlation analysis along the measurement time domain with subsequent combination of the correlation maps obtained at different 
echo times, several processing options, including simple averaging and weighted averaging of correlation maps are available. Simple averaging is used here to illustrate the enhancement of the CNR. The analysis is based on analytical expressions and numerical computations reflecting our measurement conditions.

Let $\xi\left(T E_{n}\right)$ be the correlation coefficient measured with the $n$-th echo:

$$
\xi\left(T E_{n}\right)=\frac{\sum_{r} s\left(t_{r}, T E_{n}\right) p\left(t_{t}\right)}{\bar{s}\left(T E_{n}\right) \cdot \bar{p} \cdot M} .
$$

where $s=S-\bar{S}$ and $p$ are the changes in the signal and the reference vectors, respectively, with the mean values (obtained by averaging over $I$ ) subtracted, $\bar{s}^{2}\left(T E_{n}\right) \equiv \Sigma_{r} s^{2}\left(t_{r}, T E_{n}\right) / M$, and $\bar{p}^{2} \equiv \Sigma_{r} p_{r}^{2} / M$. The correlation coefficient $\xi$ (Eq. [20]) obtained from a given voxel is assumed to vary between different fMRI experiments due to the noise contributions $g_{m}$ and $h_{r}$. In the first step, we compute the statistical mean $\left\langle\xi\left(T E_{n}\right)\right\rangle$ and its standard deviation $\sigma_{\xi}\left(T E_{n}\right)$ with respect to repetitions of the whole experiment by expanding $\xi\left(T E_{n}\right)$ in powers of the noise $\left(g_{r n}+h_{r}\right)$, which is considered to be a small parameter, such that its standard deviation $\sigma_{g h}=\sqrt{2 \sigma_{g}+\sigma_{h}}$ obeys the inequality $\sigma_{g h} \sqrt{s}\left(T E_{n}\right) \ll 1$. The results are:

$$
\begin{aligned}
\left\langle\xi\left(T E_{n}\right)\right\rangle \approx \xi_{0}\left[1-\frac{\sigma_{g h}^{2}}{2 \bar{s}^{2}\left(T E_{n}\right)}\right] & \text { and } \\
\sigma_{\xi} & \approx \frac{\sigma_{g h}}{\bar{s}\left(T E_{n}\right) \sqrt{M}} \sqrt{1-\xi_{0}^{2}}
\end{aligned}
$$

where $\xi_{0}$ is the correlation coefficient one would obtain in the absence of noise, which is less than one due to deviations of the modeled reference vector from the actual signal. As expected, $\left(\xi\left(T E_{n}\right)\right)$ decreases with increasing noise and peaks at $T E_{n}=\mathrm{T}_{2}^{*}$ (Fig. 3a). The next step is the computation of the averaged correlation coefficient $\xi=\Sigma_{n} \xi\left(T E_{n}\right) / N$. Because of our assumption of Gaussian noise, the squares of the standard deviations of different echo times are additive. Thus, similar to the above analysis, weighted averaging of correlation maps accounting for Eq. [21] would provide optimal functional sensitivity. For the sake of simplicity, we consider averaging with equal weights. The relative CNR as a function of the range of averaged echo times shows a maximum (Fig. 3b), similar to the results obtained by preprocessing in the echo time domain.

\section{MATERIALS AND METHODS}

\section{Data Acquisition}

Seven visual stimulation experiments were performed on three male subjects with a mean age of $27 \pm 4.4$ years (SD; age range $22-30$ years). Subjects gave written, informed consent for participation in the study, which was approved

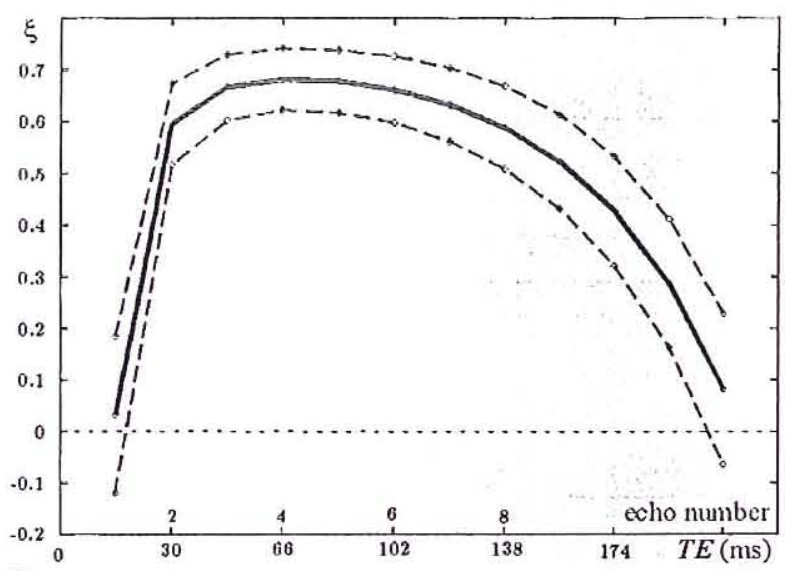

a

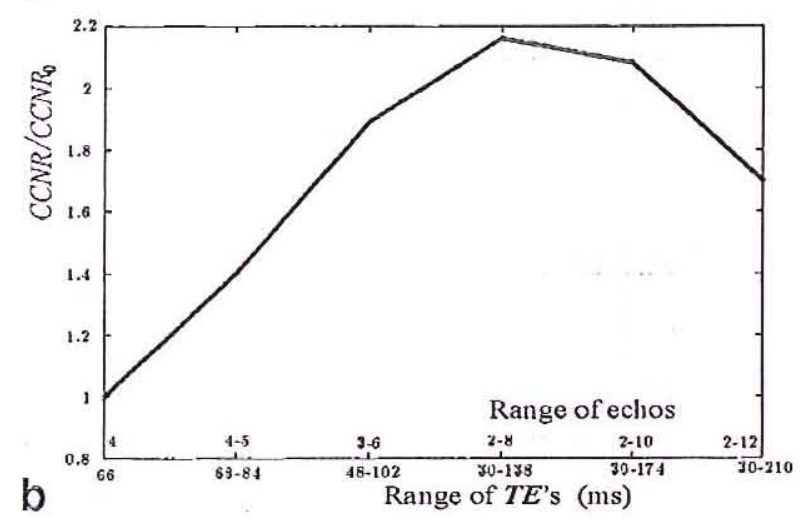

FIG. 3. a: Simulation of the mean correlation coefficient $\zeta$ and its standard deviation (dashed lines) for different echo times assuming the measurement conditions in this study (12 echoes ranging from 12 to $213 \mathrm{msec}$, echo spacing $18 \mathrm{msec}, \xi_{0}=0.8, \bar{s}\left(T E_{n}\right) / \sigma_{g+h}=5, M=$ $30)$. The results of the simulation are only qualitatively correct for $n<$ 2 and $n>10$. b: Averaging of correlation maps obtained at different echo times increases the relative contrast-to-noise ratio $\left(C_{C N R}\right)$ compared with that of the single-echo $\left(T E=T_{2}^{*}\right.$ ) correlation map $\left(\mathrm{CCNR}_{0}\right)$ depending on the range of selected echo times.

by the University of Düsseldorf Human Subjects Review Committee. Wide-angle visual stimulation with red light flashing at $8 \mathrm{~Hz}$ was applied using home-made computercontrolled LED goggles positioned directly over the subject's face. The timing of the visual stimulation was synchronized to the pulse sequence trigger of the scanner. Measurements were acquired on a Vision $1.5 \mathrm{~T}$ whole-body scanner (Siemens Medical Systems, Erlangen, Germany) equipped with a standard quadrature head coil and an EPI booster. Foam cushions to secure the head were used to reduce motion artifacts.

The turbo-PEPSI pulse sequence included lipid suppression and was designed to acquire up to 12 consecutive EPI encodings. Parameters were as follows: flip angle $90^{\circ}$; field of view (FOV) $220 \mathrm{~mm}$; matrix size $64 \times 32$ pixels; pixel size $3 \times 6 \mathrm{~mm}^{2}$; slice thickness 3 or $6 \mathrm{~mm}$; number of slices 4; and interslice spacing 0.3 or $0.6 \mathrm{~mm}$. TEs ranging from 12 to 213 msec from a single FID were used to optimize functional sensitivity, as discussed above. The duration of the trapezoidal readout gradient was $500 \mu \mathrm{sec}$, with 160 $\mu$ sec ramp times. Nonlinear sampling was employed on the 
ramps. The maximum receiver bandwidth was $196 \mathrm{kHz}$. Phase encoding was refocused between images to encode the same $k$-space trajectory in all images, resulting in a total image encoding time of $18.3 \mathrm{msec}$. The total number of images that could be acquired was dependent on selection of the TR, number of slices, and number of echoes due to software limitations.

To assess the robustness of multiecho sampling for different stimulation conditions, seven experiments using five stimulation paradigms listed in order of decreasing stimulus duration and decreasing functional CNR were performed:

Paradigm 1: $15 \mathrm{~s}$ baseline and 3 repetitions consisting of 12 $\mathrm{s}$ stimulation and $18 \mathrm{~s}$ baseline condition (TR: $3 \mathrm{~s}$ ).

Paradigm 2: $15 \mathrm{sec}$ baseline and three repetitions consisting of $9 \mathrm{sec}$ stimulation and $21 \mathrm{sec}$ baseline condition (TR 3 $\mathrm{sec}$.

Paradigm 3: $7 \mathrm{sec}$ baseline condition, $7 \mathrm{sec}$ stimulation, 13 sec baseline condition, $7 \mathrm{sec}$ stimulation, and $8 \mathrm{sec}$ baseline condition (in this experiment only eight echos were acquired; TR $1 \mathrm{sec}$ ).

Paradigm 4: $9 \mathrm{sec}$ baseline and two repetitions consisting of $6 \mathrm{sec}$ stimulation and $9 \mathrm{sec}$ baseline condition (TR 1.5 $\mathrm{sec})$.

Paradigm 5 (repeated 3 times on subject 2): 6 sec baseline and two repetitions consisting of $3 \mathrm{sec}$ stimulation and 7 sec baseline condition (TR $1 \mathrm{sec}$ ).

For comparison, the same paradigms were measured with conventional EPI (TE $66 \mathrm{msec}$ ) using the same image resolution, $\mathrm{TR}$, and readout bandwidth.

\section{Data Processing}

Turbo-PEPSI magnitude images for each echo time were reconstructed online. Data analysis was performed offline using either exponential fitting (Theory, section 3) or linear summation in the echo time domain (Theory, section 1), followed by correlation analysis in the measurement time domain to assess functional activation. Alternatively, correlation analysis was performed for each echo time separately, and the resulting correlation maps were averaged (Theory, section 4).

For quantitative relaxation mapping an exponential function:

$$
S=S_{o} e^{-T E / T_{2}^{*}}
$$

was fitted to the magnitude of the FID data of each voxel using the conjugate gradient method (20). Parametric images of $T_{2}^{*}$ and initial signal magnitude $S_{0}$ were computed from voxels where the signal intensity at the first echo time exceeded a threshold of $10 \%$ of the maximum signal amplitude. In the remainder of the voxels these parameters were set to zero.

Linear summation of turbo-PEPSI magnitude images obtained at different echo times was performed for different ranges of echo times to assess the degree of sensitivity enhancement compared with a conventional EPI and with the magnitude image of the fourth turbo-PEPSI echo (which corresponds to the EPI measurement and thus provides maximum BOLD-contrast). The following ranges of summa- tion were employed: echoes $4-5$, echoes $3-6$, echoes $1-8$, echoes 1-10, and echoes 1-12.

Images were analyzed for evidence of gross motion artifacts using a cine loop. To assess more subtle motion artifacts, images were analyzed for spurious activation at tissue edges along the perimeter of the brain. None of the above were detectable in this study. Functional activation was assessed by cross-correlation with a boxcar reference vector delayed by $3 \mathrm{sec}$ using the Stimulate software package (21).

Two regions of interest (ROIs) were drawn manually according to the brain atlas of Tallairach and Tourneaux (22). The first ROI encompassed primary visual cortex (BA 17) and adjacent extrastriate areas (BA18). The second ROI contained a nonactivated region in frontal cortex (BA 9, BA 10).

For all seven stimulation experiments, the means and standard deviations of the correlation coefficients in ROI1 were obtained as a function of the range of summed up echoes, for $\mathrm{T}_{2}^{*}$-mapping and for EPI. The means from ROI1 were transformed to the Fisher $z$-value:

$$
z=\frac{1}{2} \ln \frac{1+\xi}{1-\xi}
$$

which is expected to be approximately Gaussian distributed (20). The statistical significance of changes in $z$ transformed mean correlation coefficients in ROI1 relative to the fourth turbo-PEPSI echo was assessed as a function of the range of summed up echoes, for $\mathrm{T}_{2}^{*}$-mapping and for EPI using a paired two-tailed $t$-test. In addition, the extent of visual activation was measured as a function of the range of summed up echoes, for $\mathrm{T}_{2}^{*}$-mapping and for EPI by counting the number of voxels in which the correlation threshold exceeded 0.7 . The statistical significance of changes in extent of visual activation relative to the fourth turbo-PEPSI echo was assessed as a function of the range of summed up echoes, for $\mathrm{T}_{2}^{*}$-mapping and for EPI using a paired two-tailed $t$-test.

To compare the specificity and sensitivity of turbo-PEPSI data summed up over 12 echos with EPI, the distributions of correlation coefficients in ROI1 and ROI2 from one of the experiments (subject 1, paradigm1) were compared using a Wilcoxon signed rank test. To characterize noise in this experiment, the standard deviation of the signal in individual pixels in ROI2 and outside of the brain was measured in the domain of the repetition time as a function of echo time. For spatially uniform white Gaussian noise in magnitude images, it is expected that the standard deviation inside the brain is $66 \%$ larger than outside the brain due to the computation of the magnitude.

\section{RESULTS}

Functional signal changes in response to visual stimulation, which were detectable in all experiments, increased in amplitude and in extent with increasing stimulus duration. Using a stimulus duration between 7 and $12 \mathrm{sec}$ (paradigms 1-3), extensive areas of activation encompassing primary visual cortex (V1) and adjacent extrastriate areas were measured. When comparing EPI and turbo- 
1

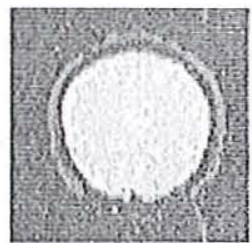

5

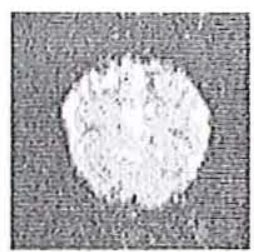

9

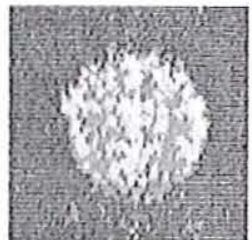

2

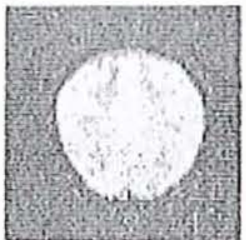

6

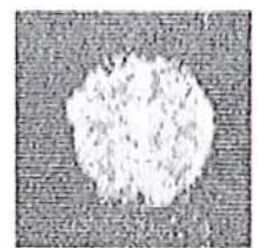

10

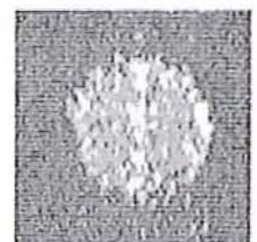

3

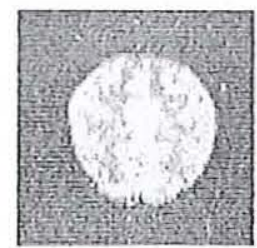

4

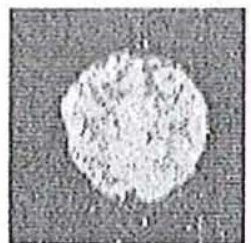

7

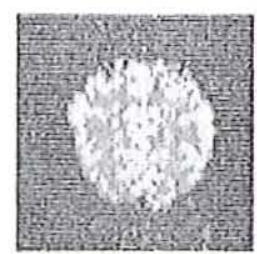

8

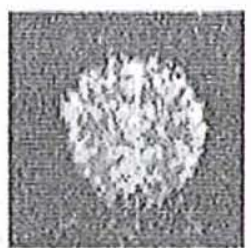

11

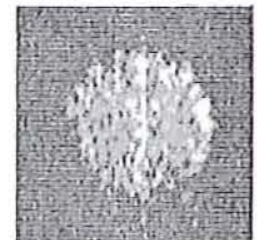

12

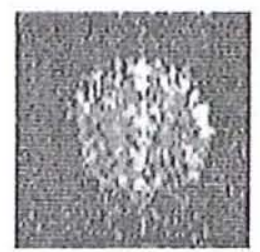

FIG. 4. Typical turbo-PEPSI correlation maps of visual activation obtained at 12 consecutive echo times ranging from 12 to 213 msec. Results from the second of the four measured slices (see Fig. 5) are shown (TR $3 \mathrm{sec}$, voxel size $3 \times 6 \times 6 \mathrm{~mm}^{3}$, correlation threshold 0.4). Activation can be detected at all echo times. Maximum sensitivity is obtained at the fourth and fifth echo times.

PEPSI images obtained at a TE of $72.5 \mathrm{msec}$ (fourth echo), very similar patterns of activation were detected. The observed changes in functional signal amplitude measured with turbo-PEPSI ranged from 3 to $20 \%$ depending on the echo time, on the anatomical location, and on the paradigm. As expected, the strength and extent of the activation measured with turbo-PEPSI as a function of echo time had a broad maximum in the vicinity of $\mathrm{TE} \approx \mathrm{T}_{2}^{*}$, but smaller signal changes could be detected at echo times as early as $12 \mathrm{msec}$ and as late as $213 \mathrm{msec}$ (Fig. 4).

The standard deviations of signal fluctuations in individual pixels in ROI2 in frontal cortex measured at different echo times did not significantly depend on echo time, consistent with our assumption that white noise domi-
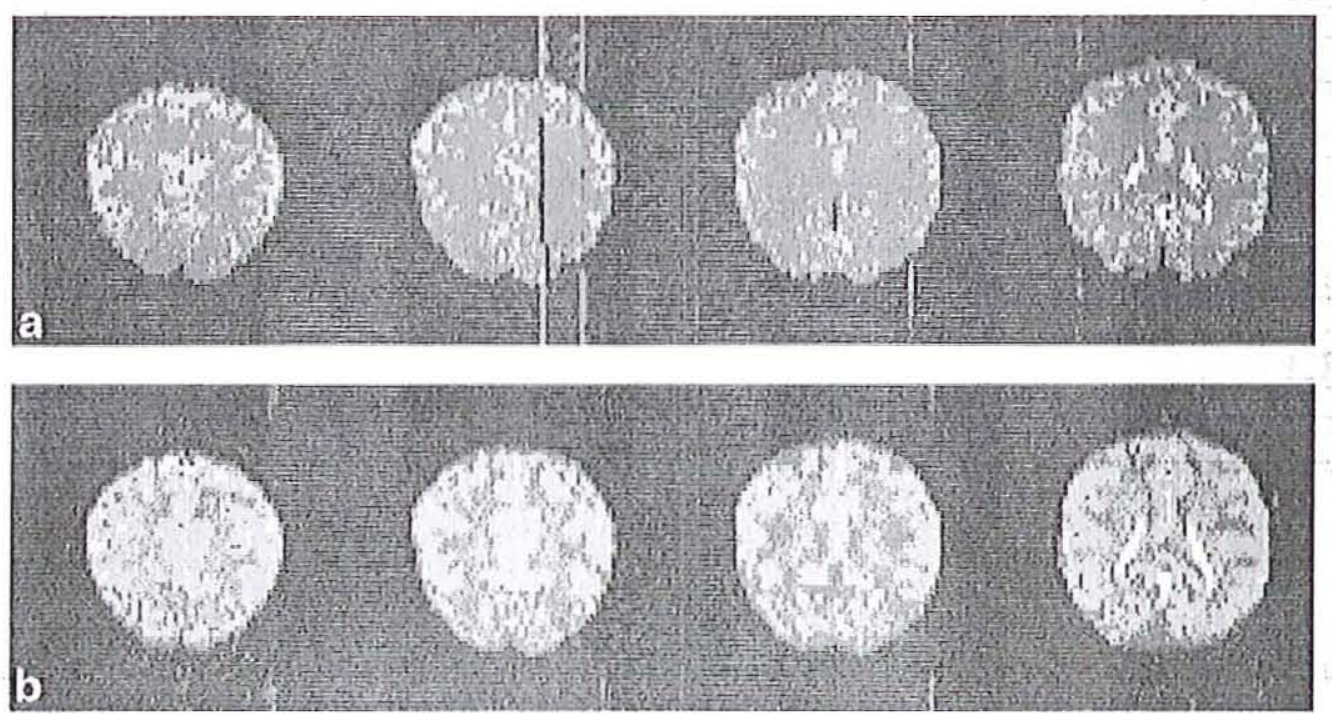

FIG. 5. Averaging turbo-PEPSI correlation maps obtained at 12 different echo times (b) enhances functional sensitivity compared with EPI (a) by reducing correlation noise (same data set as in Fig. 4, correlation threshold: 0.2 ). Vertical stripes in the EPI image are due to phase correction errors in that measurement. 
nated signal fluctuations in our data. The standard deviations in frontal cortex were about $58 \%$ larger than those measured in pixels outside of the brain, consistent with our estimate for white Gaussian noise described above.

To demonstrate the sensitivity enhancement achievable with multiecho sampling. Fig. 5 displays a comparison between EPI and the average of the turbo-PEPSI correlation maps obtained at different echo times. The strong reduction of the correlation noise in the averaged map clearly improves the delineation of the activated area.

Using echo summation, the mean correlation coefficients over ROI1 in visual cortex and the spatial extent of activation increased strongly with increasing number of summed up echoes (Fig. 6). For paradigms 1-3, images summed up over the whole range of echo times displayed similar mean correlation coefficients and extents of areas
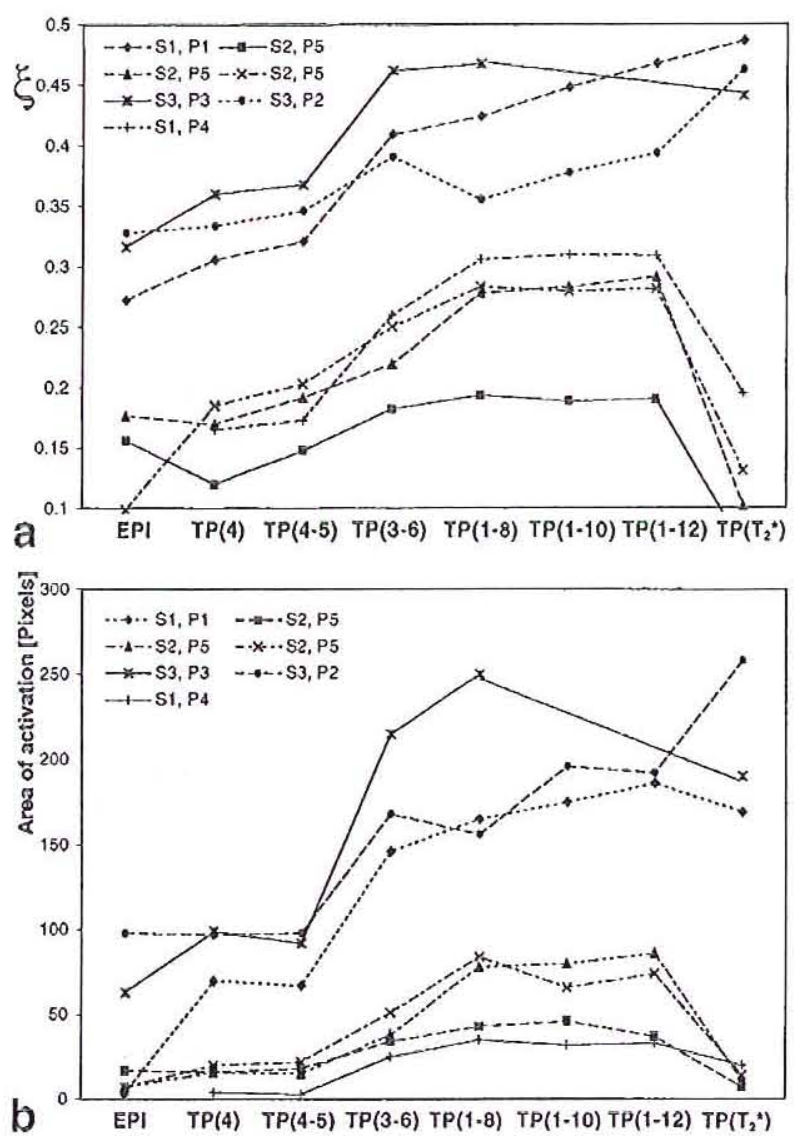

FIG. 6. Comparison of mean cross-correlation coefficients $(\xi)$ in a manually defined ROI in visual cortex (a) and extent of activation (b) (number of activated pixels in visual cortex and adjacent areas with a minimum correlation threshold of 0.7 ) obtained with EPI, with turboPEPSI for different ranges of summed-up echo times (TP4 ... TP1-12), and by using the $T_{2}^{\star}$ maps from the same turbo-PEPSI data $\left[\mathrm{TP}\left(\mathrm{T}_{2}^{*}\right)\right]$. Seven measurements were obtained in three subjects (S1-S3) using visual stimulation paradigms (P1-P5) with different stimulus duration (decreasing from $\mathrm{P} 1$ to $\mathrm{P} 5$ ) to assess the robustness of the technique for different stimulation conditions. The mean correlation coefficients and the extent of activation increase strongly with the number of summed up echoes and the duration of the stimulus (P5-P1). The lines are plotted as a guide to the eye. of activation as $\mathrm{T}_{2}^{*}$-images obtained with curve fitting (Figs. 6, 7). However, our least squares fitting seemed to be less robust than echo summation for the smaller $\mathrm{T}_{2}^{*}$ changes associated with paradigms 4 and 5 , resulting in smaller extents of activation and mean correlation coefficients. In all experiments, $S_{0}$ images showed only minor activation-related signal changes, even at a TR of $1 \mathrm{sec}$, suggesting that inflow effects were small.

Table 1 summarizes the data shown in Fig. 6. The increase in $z$-transformed mean correlation coefficient in visual cortex with echo summation was statistically significant $(P<0.002)$ for all ranges of summed up echoes. The increase in extent of activation was statistically significant $(P<0.05)$ for all ranges of summed up echoes. The major gain in sensitivity was obtained when summing up eight echoes. Extending the range of summation to later echoes resulted in smaller increases, which indicates that the gain in sensitivity reaches a maximum. This dependence on the number of summed up echoes is consistent with the shape of the corresponding theoretical CNR curve shown in Fig. 2 , which for our measurement parameters reaches its maximum at 12 echoes. By contrast, when averaged over all experiments, $\mathrm{T}_{2}^{*}$-mapping does not significantly increase the mean correlation coefficient and the extent of activation compared with the fourth echo image $(P>0.1)$. This is due to the small mean correlation coefficients and extents of activation obtained with paradigms 4 and 5 . As expected, EPI yields results that are not significantly different from those of the fourth echo image $(P>0.1)$.

When comparing the specificity and sensitivity of turboPEPSI data summed up over 12 echoes with EPI, correlation coefficients in ROI1 were significantly larger with turbo-PEPSI than with EPI $(P<0.001)$. In contrast, no statistically significant difference in the reference ROI2 in frontal cortex $(P<0.5)$ were observed, indicating that there were no differences in the false-positive rate.

\section{DISCUSSION}

In this study, a statistically significant increase in functional sensitivity was demonstrated in vivo by combining functional EPI data obtained in a single shot at multiple echo times. Our analytical theory, which is based on the assumption that white noise dominates functional signal fluctuations, provides a quantitative comparison of the expected gain in sensitivity for different ranges of combined echoes and for different data-processing methods. As shown above, our in vivo data are consistent with this assumption. A qualitative agreement was shown between the experimentally determined gain in sensitivity with increasing range of summed echoes and the theory that predicts a maximum in sensitivity when summing echo times up to $3.2 \mathrm{~T}_{2}^{*}$. The gain in sensitivity compared with conventional single-echo EPI techniques increases with decreasing duration of the image encoding time $\Delta t$ (increasing receiver bandwidth), since this increases the number of echoes that can be acquired. Conversely, when significant relaxation occurs during image encoding (e.g., very high spatial resolution single-shot EPI), a multiecho acquisition may be less advantageous. Several computationally efficient methods to combine data were assessed. Summation 

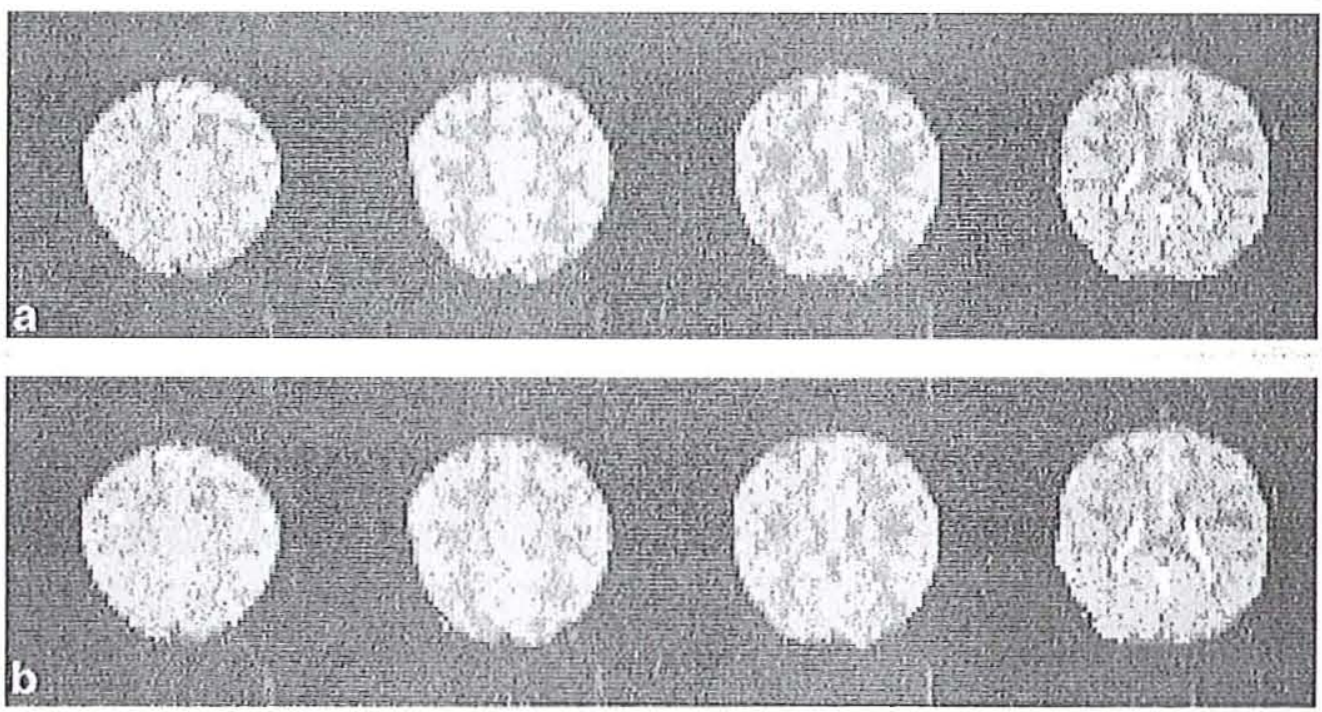

FIG. 7. Typical turbo-PEPSI correlation maps demonstrating similar extent of activation obtained by fitting (a) and by echo summation (b) (correlation threshold 0.4 ; same data sets as in Figs. 4 and 5)

of magnitude images obtained at different echo times was shown to be more robust than our curve fitting. However, curve fitting remains advantageous, since it permits quantitation of functional relaxation time changes and distinction from changes in initial signal amplitude.

Thermal noise becomes an important source of signal fluctuations at clinical field strengths when high receiver bandwidths and/or high spatial resolutions are employed. The former is advantageous to reduce susceptibilityrelated image distortions and ghosting associated with the EPI technique. Although lower readout bandwidths in combination with post-processing methods to compensate for the resulting image artifacts can be used to reduce thermal noise, significant relaxation distortions of the point-spread function that are difficult to compensate (23) remain. Our multiecho approach encourages the use of high receiver bandwidths without sacrificing functional sensitivity. The ability to distinguish changes in initial signal intensity from changes in relaxation makes singleshot multiecho sampling particularly advantageous for electrocardiographic (ECG)-gated fMRI (24-26), which is sensitive to heart-rate related fluctuations in steady-state magnetization.

A disadvantage of the technique is the increased measurement time per slice, which is approximately doubled with respect to conventional EPI. Further disadvantages include increased demands on the gradient hardware, increased eddy current effects, increased gradient noise, and possible peripheral nerve stimulation effects. Neither significant eddy current effects nor stimulation effects were experienced in this study. When scanning large volumes, multiecho sampling should preferentially be combined with

Table 1

Sensitivity Comparison Between Turbo-PEPSI Using Different Data Analysis Methods and EPI*

\begin{tabular}{|c|c|c|c|c|c|c|}
\hline Method ${ }^{a}$ & $\begin{array}{c}\text { Fisher } \\
\text { z-transformed } \\
\text { correlation coefficient } \\
z(\xi)^{b}(S D)\end{array}$ & $\begin{array}{c}\text { Relative increase of } z(\xi) \\
\text { with respect to TP4 } \\
{[\%(S D)]}\end{array}$ & $P$ value ${ }^{c}$ & $\begin{array}{l}\text { No. of activated } \\
\text { pixels }(N)^{d}(S D)\end{array}$ & $\begin{array}{c}\text { Relative increase of } N \\
\text { with respect to TP4 } \\
\text { [\% (SD)] }\end{array}$ & $P$ value ${ }^{e}$ \\
\hline TP4 & $0.24(0.10)$ & & & $46(41)$ & & \\
\hline TP4-5 & $0.26(0.10)$ & $9(7)$ & 0.0008 & $45(40)$ & $-3(13)$ & 0.0442 \\
\hline TP3-6 & $0.32(0.12)$ & $38(14)$ & 0.0003 & $97(78)$ & $176(156)$ & 0.0118 \\
\hline TP1-8 & $0.35(0.11)$ & $51(26)$ & 0.0007 & $116(78)$ & $286(243)$ & 0.0048 \\
\hline TP1-10 & $0.33(0.10)$ & 57 (25) & 0.0020 & $99(69)$ & $295(223)$ & 0.0063 \\
\hline TP1-12 & $0.34(0.11)$ & $60(24)$ & 0.0017 & $101(71)$ & $304(240)$ & 0.0082 \\
\hline $\mathrm{TP}\left(\mathrm{T}_{2}^{*}\right)$ & $0.28(0.21)$ & $6(44)$ & 0.3272 & $96(107)$ & $97(161)$ & 0.0988 \\
\hline EPI & $0.23(0.10)$ & $-6(26)$ & 0.2676 & $33(39)$ & $-41(40)$ & 0.1168 \\
\hline
\end{tabular}

Results are the average of seven experiments (see Materials and Methods and Fig. 6). SD, standard deviation.

aTP, turbo-PEPSI; TP4, . . , TP1-12, range of summed-up echoes; TP(T2), $\mathrm{T}_{2}^{*}$ map; EPI, conventional echoplanar imaging.

${ }^{b} z$-Transformed mean correlation coefficient in ROI1 in visual cortex.

'Statistical significance of changes in $z(\xi)$ given in the second column with respect to TP4 using a paired two-tailed $t$-test.

${ }^{d}$ Number of activated pixels in visual cortex and adjacent areas with a minimum correlation threshold of 0.7 .

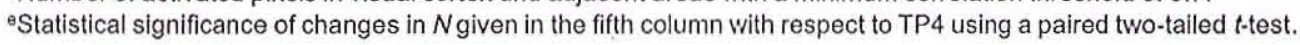


phase encoding in the third spatial dimension, since this approach provides a better signal-to-noise ratio per unit time than multislice techniques.

The optimization approach developed in this paper has important implications for comparing functional sensitivity at different magnetic field strengths and between regions with different $T_{2}^{*}$ values. Using the same single-echo acquisition technique at different field strengths may not be optimal according to our findings. For increasing $\mathrm{T}_{2}^{*}$ values [decreasing magnetic field strength (8)] an increasing number of echo times should be measured, to obtain optimum sensitivity (assuming equal gradient performance at different field strengths). However, advantages at higher magnetic field strengths remain, since the shorter $\mathrm{T}_{2}^{*}$ values require fewer echo times to be measured, enabling enhanced functional time resolution (finer sampling in the repetition time domain or more slices per unit time) compared with lower magnetic field strengths. In regions having different magnetic field inhomogeneities, multiecho sampling allows selection of optimum dataprocessing parameters in each region to maximize functional sensitivity.

The methodological approach and supporting theory developed here are also applicable to phase-encoded spectroscopic imaging, such as PEPSI, and to conventional gradient-echo techniques, which are typically measured with low-readout bandwidths, thus sampling a significant part of the relaxation curve. This explains why conventional low-bandwidth gradient-echo techniques compare favorably with EPI in terms of functional sensitivity. However, such techniques are sensitive to phase-encoding artifacts due to head motion and physiological variability. Our own experience with PEPSI indicates that single-shot spectroscopic imaging techniques are more robust than multishot techniques (9).

Considerable progress with modeling the BOLD-contrast signal relaxation using Monte Carlo simulations and analytical approaches $(27-34)$ has stimulated the development of methodology to quantitate functional signal changes in terms of physiological parameters, such as blood flow, blood volume, and blood oxygenation. Recently, it has been suggested that measuring the entire relaxation curve may allow quantitation of biophysical parameters such as the blood volume fraction and blood oxygenation (31). High-speed spectroscopic imaging offers a sensitive and robust approach for quantitating functional changes in $T_{2}^{*}$ with high temporal resolution. The choice between phaseencoded techniques such as PEPSI and single-shot techniques such as turbo-PEPSI allows flexible tradeoffs between temporal resolution in the measurement repetitiontime domain and in the echo-time domain.

The gain in sensitivity with multiecho sampling is particularly advantageous for the small signal changes seen in single-event-related fMRI and for real-time fMRI, which is currently under development in our laboratory (35-37). The possibility of summing up echo signals facilitates on-the-fly processing in real-time, which is not feasible with fitting. We are currently using this approach to map brain activity in single-trial experiments in real time. Although echo summation yields qualitative functional maps, it is robust even for small changes in $\mathrm{T}_{2}^{*}$ and thus provides information that is supplementary to that obtained by fitting.

In conclusion, maximum functional sensitivity at any magnetic field strength and for regional variations in magnetic field inhomogeneity can be achieved by multiecho sampling of relaxation signals. Single-shot spectroscopic imaging techniques, such as turbo-PEPSI, offer a data acquisition speed comparable to that of conventional EPI, as well as several distinct advantages: increased functional sensitivity, absolute quantification of changes in $\mathrm{T}_{2}^{*}$ and initial signal intensity, and reduced image distortions.

\section{ACKNOWLEDGMENTS}

We thank Michael Peyerl (Siemens Medical System, Erlangen, Germany), Michael Bock (German Cancer Research Center, Heidelberg, Germany), and N. Jon Shah (Research Center Jülich $\mathrm{GmbH}$, Germany) for helpful comments regarding pulse-sequence programming. We thank Dieter Suter (University of Dortmund, Germany) for useful discussions.

\section{REFERENCES}

1. Menon RS, Ogawa S, Tank DW, Ugurbil K. 4 Tesla gradient recalled echo characteristics of photic stimulation-induced signal changes in the human primary visual cortex. Magn Reson Med 1993;30:380-386.

2. Bandettini PA, Wong EC, Jesmanowicz A, Hinks RS, Hyde JS. Spin-echo and gradient-echo EPI of human brain activation using BOLD contrast: a comparative study at 1.5T. NMR Biomed 1994;7:12-20.

3. Kirsch JE, Chen Q, Andersen A, Blonder XL, Smith C, Avison M. Functional MR imaging with multiple GRE signal intensities. Radiology 1994;193(suppl):abstract 93.

4. Andersen AH, Kirsch JE, Chen Q. Avison MJ. Data analysis for multi-echo acquisition in fMRI. In: Proceedings of the SMR 2nd Annual Meeting, San Francisco, 1994. p 639.

5. Van der Veen JWC, den Hollander JA, Pohost GM. Human brain activation: perfusion or BOLD effect. In: Proceedings of the 2nd Annual Meeting, San Francisco, 1994. p 623.

6. Chen Q. Andersen AH, Avison MJ. TE-dependence of gradient echo signal decay. In: Proceedings of the ISMRM 4th Annual Meeting. New York, 1996. p 290.

7. Chen Q, Andersen AH, Zhang Z, Ovadia A, Gash DM, Avison MJ. Mapping drug-induced changes in cerebral $R_{2}^{*}$ by multiple gradient recalled echo functional MRI. Magn Reson Imaging 1996:14:469-476.

8. Gati ], Menon RS, Ugurbil K, Rutt BK. Experimental determination of the BOLD field strength dependence in vessels and tissue. Magn Reson Med 1997;38:296-302

9. Posse S, Wiese S, Behr D, Grosse-Ruyken ML, Shah NJ, Richards T, Dager S, Müller-Gärtner H-W. Detection of brain activation by oxygenation sensitive proton-echo-planar-spectroscopic-imaging (PEPSI). In: Proceedings of the ISMRM 5th Annual Meeting, Vancouver, BC, 1997. p 376.

10. Bandettini PA, Kwong K, Davis TL, Tootell RBH, Wong EC, Fox PT Belliveau JW, Weiskoff RM, Rosen BR. Characterization of cerebral blood oxygenation and flow changes during prolonged brain activation. Hum Brain Mapping 1997:5:93-109.

11. Clare $S$, Bowtell R, Morris P. Single shot $T_{2}^{*}$ measurement during visual and motor activation. MAGMA 1997:5(suppl):abstract 329.

12. Posse S, Wiese S, Kessler C, Gembris D, Weiss U, Peyerl M, GrosseRuyken ML. Elghawaghi B, Richards T, Dager S. Single shot $\mathrm{T}_{2}^{*}$. sensitive spectroscopic imaging increases $\mathrm{MRI}$ sensitivity: preliminary evidence from visual and olfactory activation. In: Proceedings of the ISMRM 6th Annual Meeting, Sydney, 1998. p 299.

13. Speck $\mathrm{O}$, Hennig $\mathrm{J}$. Functional imaging by $\mathrm{I}_{0}$ and $\mathrm{T}_{2}^{*}$ parameter mapping using multi-image EPI. Magn Reson Med 1998;40:243-248.

14. Posse S, Wiese S, Kessler C, Gembris D, Weiss U, Peyerl M, GrosseRuyken ML, Elghawaghi B, Richards T, Dager S. Functional MR imaging of visual and olfactory activation using quantitative $\mathrm{T}_{2}^{*}$-mapping. Neuroimage 1998;7:445. 
15. Posse S, DeCarll CS, LeBihan D. 3D echo-planar MR spectroscopic imaging at short echo times in human brain. Radiology 1994;192:733738.

16. Posse S, Tedeschi G, Risinger R, Ogg R, LeBihan D. High speed 'H spectroscopic imaging. Magn Reson Med 1995:33:34-40.

17. Hennig J, Ernst T, Speck O, Deutschl G, Feifel E. Detection of brain activation using oxygenation sensitive functional spectroscopy. Magn Reson Med 1994:31:81-84.

18. Ernst T, Hennig J. Observation of a fast response in functional MR. Magn Reson Med 1994:32:146-149.

19. Biswal B, Yetkin F, Haughton V, Hyde J. Functional connectivity in the motor cortex of resting human brain using echo-planar MRI. Magn Reson Med 1995;34:537-541.

20. Press WH, Teukolsky SA, Vetterling WT, Flannery BP. Numerical recipes in $\mathrm{C}$ : the art of scientific computing. Cambridge: Cambridge University Press; 1992.

21. Strupp JP. Stimulate: a GUI based fMRI analysis software package. Neuroimage 1996;3:S607.

22. Talairach J, Tournaux P. Coplanar stereotaxic atlas of the human brain. New York: Thieme Medical; 1988.

23. Haacke EM. The effects of finite sampling in spin-echo or field-echo magnetic resonance imaging. Magn Reson Med 1987:4:407-421.

24. Guimaraes AR, Baker JR, Weisskoff RM. Cardiac-gated functional MRI with $\mathrm{T}_{1}$ correction. In: Proceedings of the ISMRM 3rd Annual Meeting, Nice, 1995. p 798.

25. Posse S, Cheng G-Q, Müller-Gärtner H-W, Shah NJ. Increased functional contrast in functional echo planar imaging by EKG.gating. In: Proceedings of the ISMRM 4th Annual Meeting. New York, 1996. p 1837.

26. Kuppusamy K, Lin W, Haacke EM. Statistical assessment of crosscorrelation and variance methods and the importance of electrocardiogram gating in functional magnetic resonance imaging. Magn Reson Imaging 1997;15:169-181.

27. Ogawa S, Menon RS, Tank DIV, Kim S.G, Merkle H, Ellerman JM Ugurbil K. Functional brain mapping by blood oxygenation level dependent contrast magnetic resonance imaging. Biophys J 1993;64:803812.
28. Weisskoff RM, Zuo CZ, Boxerman JL, Rosen BR. Microscopic susceptibility variation and tranverse relaxation: theory and experiment. Magn Reson Med 1994;31:601-610.

29. Yablonskiy DA, Haacke EM. Theory of NMR signal behavior in magnetically inhomogeneous tissue: the static dephasing regime. Magn Reson Med 1994;32:749-763.

30. Boxerman JL. Hamberg LM, Rosen BR. Weisskoff RM. MR contrast due to intravascular magnetic susceptibility perturbations. Magn Reson Med 1995;34:555-556.

31. Yablonskiy DA, Haacke EM. An fMRI method for measuring $T_{2}$ in the presence of static and RF magnetic field inhomogeneities. Magn Reson Med 1997;37:872-876.

32. Kiselev VG, Posse S. Analytical model of susceptibility induced MR signal dephasing: effect of diffusion in a microvascular network. Magn Reson Med 1999:41:499-509.

33. Kiselev VG, Posse S. Theory of susceptibility induced NMR signal dephasing in a cerebrovascular network. Phys Rev Lett 1999;81:56965699.

34. van Zijl PCM, Eleff SM, Ulatowski JA, Oja JME, Ulug AM, Traystman RJ. Kauppinen RA. Quantitative assessment of blood flow, blood volume and blood oxygenation effects in functional magnetic resonance imaging. Nature Med 1998;4:159-167.

35. Posse S, Schor S, Gembris D, Müller E, Peyerl M, Kroeker R, GrosseRuyken ML, Elghawaghi B, Taylor JG. Real-time fMRI on a clinical whole body scanner: single trial detection of sensorimotor stimulation and visual recall activation. In: Proceedings of the ISMRM 6th Annual Meeting, Sydney, 1998. p 162.

36. Schor S, Gembris D, Taylor JG, Peyerl M, Müller E, Posse S. Functional imaging in real-time (FIRE). In: Proceedings of the ISMRM 6th Annual Meeting, Sydney, 1998. p 1440.

37. Gembris D, Taylor JG, Schor S, Kiselev V, Suter D, Posse S. Methodology of fast correlation analysis for real-time fMRI experiments. In: Proceedings of the ISMRM 6th Annual Meeting. Sydney, 1998. p. 1486. 